This item was submitted to Loughborough's Research Repository by the author.

Items in Figshare are protected by copyright, with all rights reserved, unless otherwise indicated.

\title{
Genetic evolution of sorting programs through a novel genotype-phenotype mapping
}

PLEASE CITE THE PUBLISHED VERSION

http://www.insticc.org/InsticcPortal/Home.aspx

PUBLISHER

INSTICC

VERSION

AM (Accepted Manuscript)

LICENCE

CC BY-NC-ND 4.0

\section{REPOSITORY RECORD}

Xhemali, Daniela, Chris J. Hinde, and R.G. Stone. 2019. "Genetic Evolution of Sorting Programs Through a Novel Genotype-phenotype Mapping”. figshare. https://hdl.handle.net/2134/7342. 
This item was submitted to Loughborough's Institutional Repository (https://dspace.lboro.ac.uk/) by the author and is made available under the following Creative Commons Licence conditions.

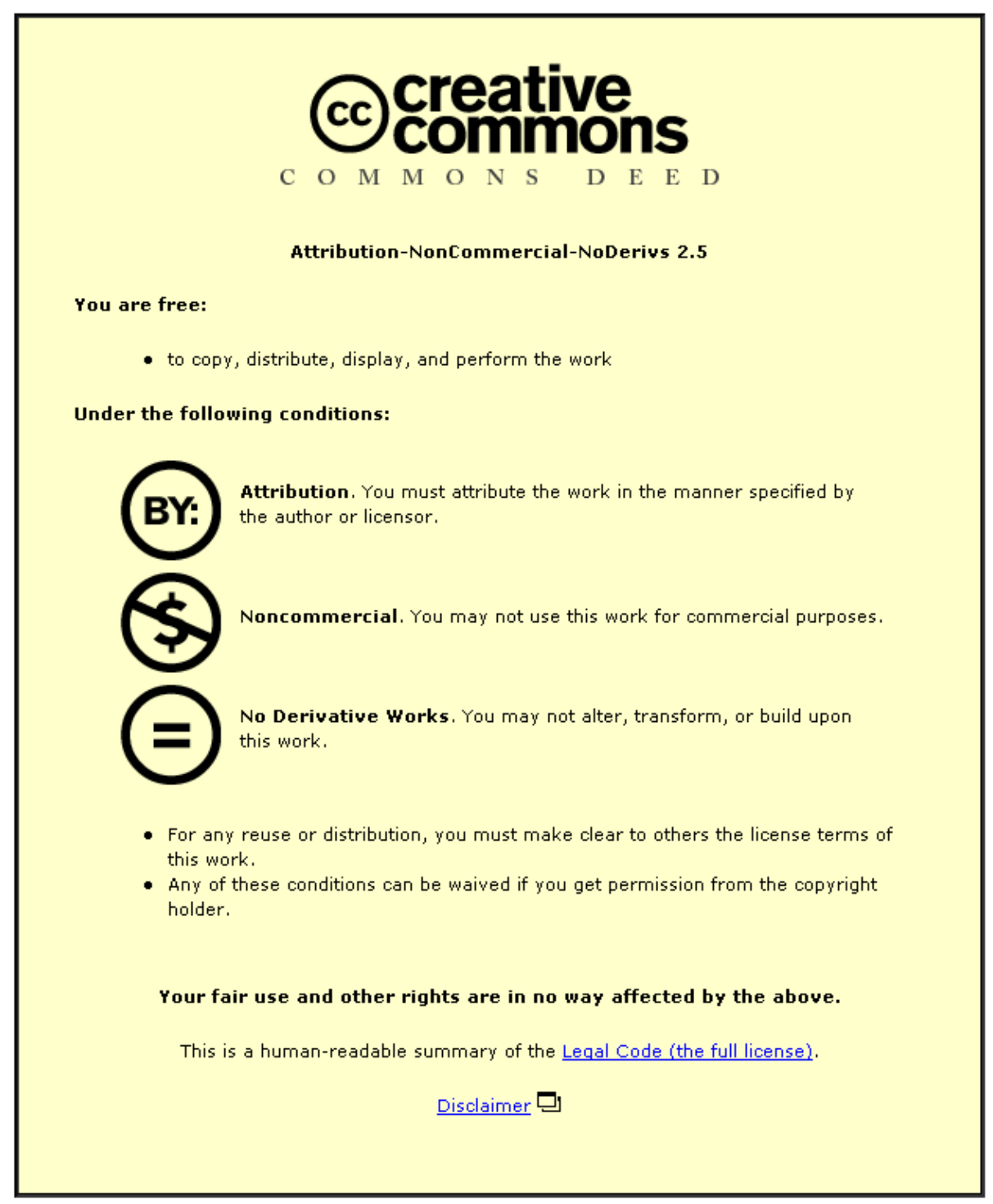

For the full text of this licence, please go to: http://creativecommons.org/licenses/by-nc-nd/2.5/ 


\title{
GENETIC EVOLUTION OF 'SORTING' PROGRAMS THROUGH A NOVEL GENOTYPE-PHENOTYPE MAPPING
}

\author{
Daniela Xhemali, Christopher J. Hinde, Roger G. Stone \\ Department of Computer Science, Loughborough University, Loughborough, United Kingdom \\ D.Xhemali@lboro.ac.uk,C.J.Hinde@lboro.ac.uk,R.G.Stone@lboro.ac.uk
}

Keywords: Genetic Programming, Genotype-Phenotype mapping, XML, Regular Expressions, Software Programs.

\begin{abstract}
This paper presents an adaptable genetic evolutionary system, which includes an innovative approach to mapping genotypes to phenotypes through XML rules. The evolutionary system was originally created to evolve Regular Expressions (REs) to automate the extraction of web information. However, the system has been adapted to work with a completely different domain - Complete Software Programs - to demonstrate the flexibility of this approach. Specifically, the paper concentrates on the evolution of 'Sorting' programs Experiments show that our evolutionary system is successful and can be adapted to work for challenging domains with minimum effort.
\end{abstract}

\section{INTRODUCTION}

Genetic Programming (GP) can be defined as " $a$ systematic, domain-independent method for getting computers to automatically solve problems starting from a high-level statement of what needs to be done" (Langdon et al., 2008). GP research has attracted attention in various fields such as: game strategies (Keaveney \& O'Riordan, 2009), military defence (Jackson, 2005), plant biology (Dyer \& Bentley, 2002), electronics (O'Neill et al., 2001), railway platform allocation (Clarke et al., 2009), spam filtering (Conrad, 2007), feature extraction from media files (Hsu, 2007; Klank et al., 2008) etc.

An area that has also managed to secure the attention of GP is the automation of Web Information Extraction (WIE) (Atkinson-Abutridy et al., 2004; Barrero et al., 2009; Xhemali et al., 2010b). The research presented in this paper was originally set up to evolve Regular Expressions (REs) to automate the extraction of web information, specifically training course information such as: course names, dates, locations and prices. The details of this part of the research, including experimental results, were covered previously (Xhemali et al., 2010-b), thus they will not be covered again in this paper.

This paper focuses on a specific part of GP - the genotype to phenotype mapping. Previous work (Xhemali et al., 2010-a) gave details of our innovative approach in relation to its application to REs and to Complete Software Statements (Withall et al., 2008) such as FOR loops, IF statements etc. This paper concentrates on the genotype to phenotype mapping process in relation to Complete Software Programs. Specifically, experiments are carried out to test the complete GP process for the evolution of 'Sorting' programs. 'Sorting' programs were chosen in order to demonstrate the flexibility of this approach, as such programs represent an entirely different domain to REs and a challenging problem for GP systems.

\section{RELATED RESEARCH}

The genotype-phenotype mapping relates to the way individuals in a population are represented, as this can have a significant effect on the performance of GP. A genotype represents each individual in the search space, whereas its phenotype represents the individual in the solution space (Banzhaf, 1994). Some research, particularly earlier GP research, does not make a distinction between genotypes and phenotypes (Koza, 1992; Whigham, 1995; Conrad, 2007, Snajder et al., 2008 etc.). Individuals in each genetic population remain the same throughout the evolution process. In these works the search space and the solution space are identical.

In 1994, Banzhaf suggested the separation of the two spaces and introduced his work on the genotype-phenotype mapping. The separation involves the encoding of the individuals to a form 
known as the genotype, which is later on decoded back to the corresponding program, referred to as the phenotype. Since then, many other researchers have embraced the separation into genotypes and phenotypes (Keller \& Banzhaf, 1996; Withall et al., 2008; Clarke et al., 2009 etc.). This separation simplifies and increases the efficiency of certain genetic operations such as: reproduction and mutation, because these would no longer be constrained by the parameters used in the program being evolved. In genotype-phenotype based GP, genetic operators such as Crossover and Mutation would be performed on the genotype, whereas other processes, such as the Fitness scoring, would be performed on the phenotype. Sections 3.2 and 3.3 explain this concept further through examples.

On the downside, however, this adds an additional step to the genetic evolution process - the translation or mapping of the genotypes to their corresponding phenotypes. This step occurs after the genetic reproduction stage (i.e. the crossover and mutation) and before the Fitness test can take place.

There are researchers who criticise the separation into genotypes and phenotypes (Moore, 2000). The main concern expressed is that the conversion process of a mutated genotype into the phenotype may result in anomalies that could potentially lead to invalid solutions. A direct mapping between the encoded program (genotype) and the actual program (phenotype) is therefore vital to ensure the validity of the solutions (Rothlauf, 2006; Withall et al., 2008).

The following discusses different methods that have been used to achieve the mapping process.

Banzhaf (1994; 2006) represented his genotypes as linear binary strings. The mapping stage then processed these genotypes from left to right in 5-bit sections, where each 5-bit code mapped to a prespecified symbol. For example: 00000 mapped to PLUS, 00100 mapped to POW, 11000 mapped to variable $X$ etc. The first bit indicated whether the code represented a function (PLUS, POW etc.) or a terminal (X, Y etc.). The research also discussed their concern about generating constant numbers. Koza (1992) had solved this problem by defining "random ephemeral constants" where constants are only generated once for a particular program and then reused wherever they are needed within that program.

Keller (1996) continued in the footsteps of Banzhaf, concentrating on providing experimental evidence for choosing the genotype-phenotype approach instead of the normal GP approach. Keller used the LALR (Look Ahead LR) parser for the repairing stage of the genotype-phenotype mapping process. LALR parsers scan the input from left to right and construct a rightmost derivation in reverse (Aho and Ullman, 1979).

There was a certain amount of redundancy in the genetic coding in both Banzhaf's and Keller's works. They both admitted that, in their works, different binary strings could correspond to the same symbol, which could lead to inconsistencies e.g. 000 and 100 both mapping to ' $a$ '.

A slightly different genotype representation is seen in the work of Withall et al. (2008). In here genotypes are represented as linear blocks of integers. Each block consists of exactly four integers, each integer representing a different gene. Although both research works used fixed-length genomes, in the work of Banzhaf $(1994 ; 2006)$ the resulting phenotypes could vary in length, whereas in (Withall et al., 2008) they remained fixed. However, Withall allowed for variable-length genomes through padding, whereby shorter program structures or statements ignored outstanding genes. The first integer in Withall's genotype always determines the type of function that follows.

Grosan and Abraham (2009) worked with multichromosome genotypes. The number of chromosomes was varied. However, the number of genes per chromosome was fixed. In this research, each gene corresponded to either a terminal: $\mathrm{T}=\{\mathrm{a}$, $\mathrm{b}, \mathrm{c}, \mathrm{d}\}$ or a function: $\mathrm{F}=\{+, *\}$. A function gene also included pointers towards the function parameters to tell the system which terminals were to be manipulated by the function. Also, the first gene of the chromosome was always a terminal. This was to ensure that only syntactically correct programs are evolved. Very differently from above, Yosif et al. (2010) introduced the novel approach of adapting a support vector machine to predict phenotypes from genotype data.

Similarly to Withall et al. (2008), the genotype in our research is represented as a string of integers. There are no fixed length genomes determined however; instead the genotype can contain any number of genes. The direct mapping of these integers to the corresponding structures is achieved through an innovative approach involving XML rules. The first gene in the string determines the XML rule to be followed, which in itself guides the mapping of the rest of the genes into a valid phenotype. This is explained in detail in section 3.2.

\section{GENOTYPES to PHENOTYPES}

As previously mentioned, some details about this research, including the GP representation chosen and a thorough explanation of our novel genotypephenotype mapping approach used on the REs 
domain, was published in a previous paper (Xhemali et al., 2010-a), thus they will not be repeated here. The rest of this paper concentrates on the application of the genotype-phenotype approach to a completely different domain - that of Software Programs, specifically 'Sorting' programs - in order to illustrate the flexibility of our approach. Additionally, the fitness function for the 'Sorting' programs is presented and experimental results are discussed.

The examples in this paper are based on the work of Withall et al. (2008). They are kept as close to the original as possible in order to ensure their integrity. One main difference however, is that in Whithall's work the evolved programs were in Perl, whereas in this paper their validity is ensured against VB script. Although the evolutionary system is developed in VB.NET with a MS SQL Server database backend, VB Script was used in order to extend the application to execute each evolved program, as these are obviously not compiled into the application. The .NET framework allows for the execution of dynamic code through the System.CodeDom.Compiler and the appropriate namespaces, however, this is more complicated and slower during execution than utilising the Microsoft Script Control from VB.NET.

\section{1 'Sorting' Program}

The 'Sorting' program was chosen, because it is a popular, well known program and a standard Computer Science problem due to its higher complexity over other small software programs such as: 'Sum finder', 'Maximum value finder' etc.

The aim of a 'Sorting' program is to order a list of integers or characters in ascending order. The output of a 'Sorting' program is therefore another list, rather than a single value.

$$
\begin{aligned}
& \text { sort: } \mathbb{Z}^{*} \rightarrow \mathbb{Z}^{*} \\
& \text { pre_sort }(L) \triangleq \text { True } \\
& \text { post_sort }(L, N) \triangleq \text { bag_of }(N)=\text { bag_of }(L) \wedge \text { ascending }(N) \\
& \quad \text { where bag_of }(\prec \succ) \neq \emptyset \\
& \quad \text { bag_of }(\prec x \succ) \neq\{|x|\} \\
& \quad \text { bag_of }\left(L_{1} \curvearrowright L_{2}\right) \neq \text { bag_of }\left(L_{1}\right) \uplus \text { bag_of }\left(L_{2}\right) \\
& \text { ascending }(N) \triangleq(\forall x, y: \mathbb{Z})(x \text { before } y \text { in } N \Rightarrow x \leq y) \\
& \text { and } \quad \begin{array}{l}
x \text { before } y \text { in } N \triangleq\left(\exists N_{1}, N_{2}, N_{3}: \mathbb{Z}^{*}\right)\left(N=N_{1} \curvearrowright \prec x \succ \curvearrowright N_{2} \curvearrowright \prec y \succ \curvearrowright N_{3}\right)
\end{array}
\end{aligned}
$$

Figure 1: Specification of 'Sort' (Withall et al., 2008)
The 'Sorting' programs evolved in this research are concerned with the sorting of lists of integers. Figure 1 shows a specification of the 'Sorting' problem.

The following explains the general details behind the genotype-phenotype mapping for software structures, as well as the additional statements and genes needed for the 'Sorting' program.

\subsection{XML Mapping}

Our genotype-phenotype mapping consists of two main components: the XML rules, which guide the system through the mapping process and the Repairing function, which makes sure that the evolved programs are syntactically correct. The genotype-phenotype mapping process is as follows:

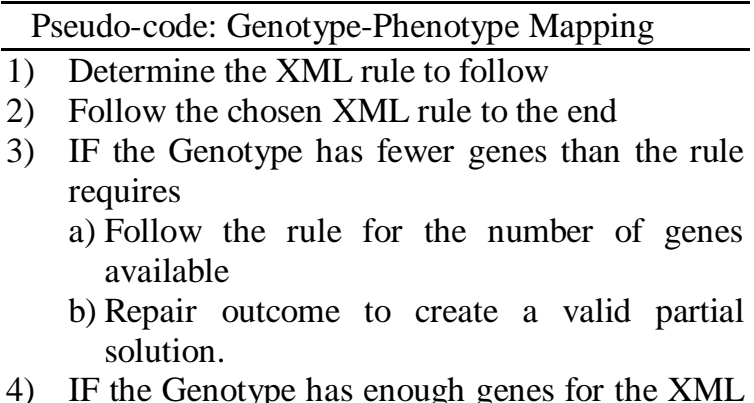

4) IF the Genotype has enough genes for the XML rule

a) Follow all the components in the rule

b) Repair outcome (if necessary) to create a valid and complete solution.

5) IF the Genotype has more genes than the rule requires

a) Follow the same steps as above ( $4 a$ and $4 b$ )

b) Ignore the rest of the genes in the Genotype

Note that this is not a character by character evolution, because this would increase the search space and dramatically increase the execution time. Instead, programs are divided into two collections: Variables (e.g. "tmp1", "tmp2", "tmp3") and Comparisons (e.g. ">”, "<", "!=" etc.). Each evolved gene is translated to an element of one of these collections. There is a separate XML rule for each software structure (e.g. "FOR", "IF ... THEN ... ELSE", “ADD”, “ASSIGN" etc.). Each rule is composed of a number of components, which guide the system through the translation of each gene to the corresponding software structure (Figure 2). For example, the IF ... THEN structure, of format (IF variable $_{1}$ comparison variable 2 THEN), requires three evolvable genes: two variables and one comparison. Table 1, Table 2 and Figure 3 illustrate 
this scenario by giving an example of the genotypephenotype mapping process.

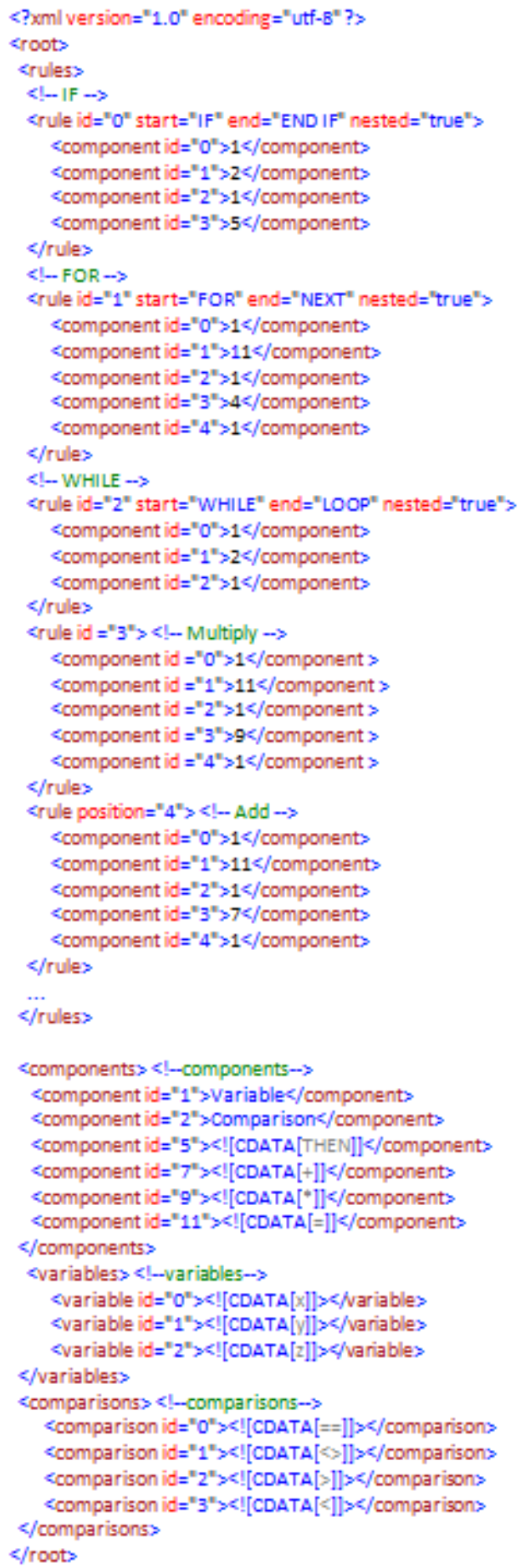

Figure 2: Sample of XML Rules

Each component refers to either the elements in the above two collections, or to other predefined elements that do not need to be evolved and as such do not require the use of any extra genes, such as: the different operators associated with each programming structure (e.g. "+" is always associated with "Add"; "=" is always associated with "Assign", thus these do not need to be evolved) or the keywords required by the programming language chosen - in this case VBScript - in order to create syntactically correct code (e.g. "THEN", “TO” etc).

The Repairing function is responsible for making sure that all the different software structures are combined correctly to create a syntactically correct and complete software program. Figure 2 shows a sample of the XML rules and components needed to guide the genotype-phenotype stage of the genetic evolution of software programs. Table 1 shows a sample Genotype to be translated using the information in Figure 2.

Note that the first gene in the genotype is always associated with the XML rule choice. The modulo function is used for this reason. In the above example (Table 1), the value of the first gene is 10 . This represents the software structure to be used. In this case, there are five different rules in the XML file, so $10 \bmod 5=0$ means that the structure chosen is an 'IF'. This structure contains four different components (Figure 2). The first three components require the use of a gene to choose from either the 'variables' or the 'comparisons' collections. The 'variables' collection has three elements, whereas the 'comparisons' has four, therefore, $27 \bmod 3=0$, $7 \bmod 4=3$ and $13 \bmod 3=1$ give elements ' $x$ ', ' $<$ ' and ' $y$ ' respectively.

The fourth component (id="5") tells the Repairing function that the THEN keyword is required next. The 'THEN' keyword is a mandatory requirement for IF statements in VB.NET or VBScript, thus this component does not need to be evolved and as such does not require the use of an extra gene from the genotype.

The nested = "true" attribute seen in Figure 2 indicates that the IF structure, differently from oneline statements, such as 'Add' or 'Multiply', expects other statement(s) inside. The following gene (gene 19) in the genotype is therefore used to determine the statement type to be nested in this IF. Therefore, $19 \bmod 5=4$ means that the next statement is 'Add'. The remaining components are dealt with in the same manner (see Table 2).

Once all the required genes have been decoded, the resulting phenotype is repaired to ensure it is syntactically correct. The Repairing function is an independent function, which scrutinises the phenotype created in order to guarantee the syntactic validity of the solution. This function is in charge of tasks like: closing software structures appropriately (e.g. FOR loops in VB.NET need to end in NEXT 
for them to be valid); adding the necessary operators, which do not need to be evolved (e.g. the 'Add' ("+") and the 'Assign' ("=") operators); tidying up the phenotype in cases when there are fewer genes available than required by the XML rule; adding header and footer information about a solution such as: variable declaration or variable return etc. All this is achieved through the use of a STACK programming structure, which works in a LIFO (Last In First Out) manner.

Figure 3 shows the complete software structure (phenotype) for the above example. The additional symbols and programming keywords added by the repairing function are shown encircled.

Table 1: Genotype

\begin{tabular}{|l|l|l|l|l|l|l|l|}
\hline 10 & 27 & 7 & 13 & 19 & 9 & 63 & 4 \\
\hline
\end{tabular}

Table 2: Genotype to Software Statement Mapping

\begin{tabular}{ccccc}
\hline $\begin{array}{c}\text { Comp. } \\
\text { No. }\end{array}$ & Component & $\begin{array}{c}\text { Gene } \\
\text { Used }\end{array}$ & Modulo & Translation \\
- & - & 10 & 0 & If \\
1 & Variable & 27 & 0 & $\mathrm{x}$ \\
2 & Comparison & 7 & 3 & $<$ \\
1 & Variable & 13 & 1 & $\mathrm{y}$ \\
5 & Then- & - & - & Then \\
- & Keyword & - & 4 & Add \\
1 & Variable & 9 & 0 & $\mathrm{x}$ \\
11 & Assign & - & - & $=$ \\
1 & Variable & 63 & 0 & $\mathrm{x}$ \\
7 & Addition & - & - & + \\
1 & Variable & 4 & 1 & $\mathrm{y}$ \\
\hline
\end{tabular}

\begin{tabular}{|l|} 
If $x<y$ Then \\
$x=x+y$ \\
End If
\end{tabular}

Figure 3: Phenotype

Updating the XML rules, once written, would require little effort, because software statements and structures are rigid in the number of components needed and the order in which they are needed. For example, the Add statement mentioned above may sum up more than two variables, however, there will always be need for one variable to which this sum is assigned, one 'Assign' operator and one or more 'Addition' operators. Adding new XML rules for new statements or structures would be equally as effortless, because it would only require the addition of the different components for that structure to the $\mathrm{XML}$ rules as well as any additional variables or comparisons that may be required.

The changes that were made to our GenotypePhenotype mapping process for the 'Sorting' software programs involved the simple addition of one more structure and a few more variables and comparison values. Specifically, a special nested FOR loop was added to the current software structures, similarly to Withall et al. (2008), which includes two nested FOR loops in the following format:

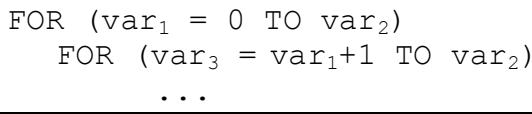

The above could have been achieved through the existing FOR loop structure (Figure 2), however, we chose to add the nested FOR loop, because this is a standard structure used when comparing elements in a list and it results in faster execution of the evolution process.

Due to the 'Sorting' program dealing with lists or arrays of integers, a few more variable types needed to be added to distinguish between normal variables and array variables (since a normal variable $\mathrm{x}$ is entirely different from the variable array(x)). This is to maintain the accuracy of the evolved solutions.

The Experimental Results section presents and discusses the results of our experiment.

\subsection{Fitness Function}

Similarly to Withall et al. (2008), the fitness function in this research has been simplified to only compare adjacent items in the list of integers rather than all the possible pairs in the list. This decision was made in order to speed up the evolution process.

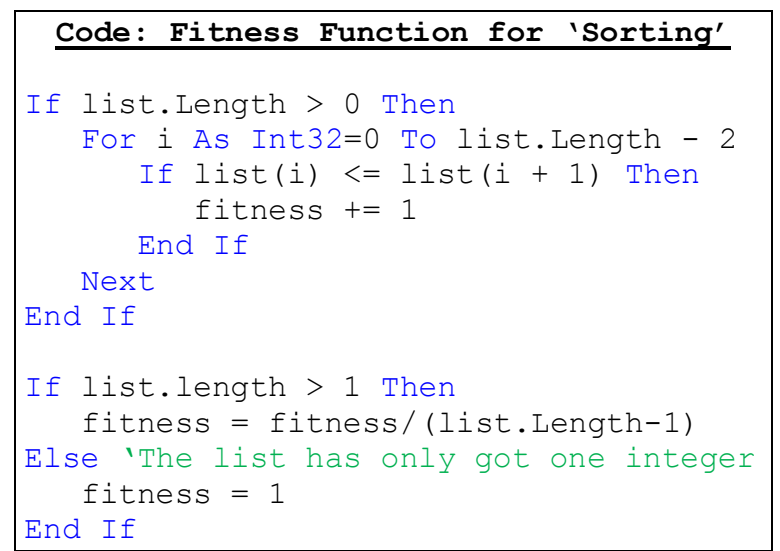


The code for the 'Sorting' program is presented above to show its simplicity. Note that the necessary header information such as variable declaration and initialisation are left out for clarity. Also note that this function is only called if the post-evolution list of integers contains the same elements as the preevolution list of integers.

\section{EXPERMIMENTAL RESULTS}

Details about the evolutionary system used in this research were discussed in a previous paper (Xhemali et al., 2010-b) however, the main parameters used by the system are summarised in here to help the reader fully appreciate the experimental results presented:

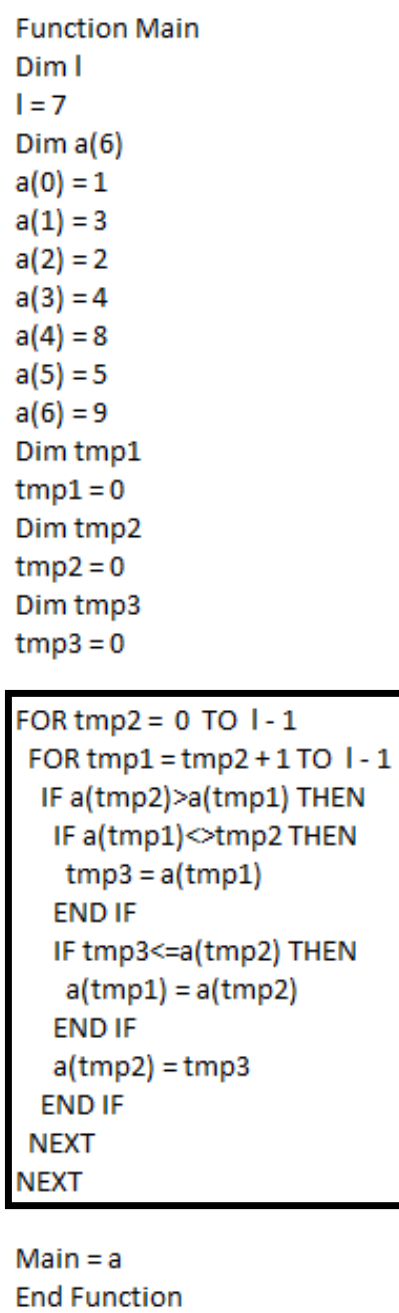

Figure 4: Complete ‘Sorting' Solution: (1)
- Population size: 10

- Tournament size: $40 \%$

- Fitness Target: 1

- Uniform Crossover Rate: $50 \%$

- Mutation Rate: 1 gene per genotype

- Initial Population: Random

The following gives the results from the experiments set up for the evolution of 'Sorting' programs. In order to maintain the similarity with the work of Withall et al. (2008), there were ten runs of the experiment, each with a maximum number of generations of 50,000. None of the experiments needed this many generations however, and each run produced a valid 'Sorting' program.

Figure 4 shows one of the 'Sorting' programs produced by the system. Note that this example shows the full VBScript code executed from within VB.NET 2008. The header and footer of the solution (shown in Figure 4 within the dotted rectangle) was added to the solution by the Repairing function. The part of the code shown within the solid rectangle was evolved by the system (and tidied up by the Repairing function, as described in section 3.2).

All experiments presented in this paper were based on the sorting process of a fixed list of seven integers $-a(1,3,2,4,8,5,9)$ - however, further experiments were carried out with different integer lists to ensure that the results in this paper were not somehow influenced by the integers chosen.

Table 3 gives information about five of the 'Sorting' solutions evolved (Fitness Score: 1), which were also generated by Withall et al. (2008). The information includes the number of generations (Gens) taken for the solution to be generated and the amount of time taken to arrive to this solution. The remaining five solutions (6-10), also with a Fitness Score of 1, refer to additional 'Sorting' solutions evolved during the experiments for this paper.

Table 3: 'Sorting' Results

\begin{tabular}{c|c|c|c|c}
$\begin{array}{c}\text { Solution } \\
\text { No. }\end{array}$ & Gens & $\begin{array}{c}\text { Gens } \\
\text { (Withall) }\end{array}$ & Time & $\begin{array}{c}\text { Time } \\
\text { (Withall) }\end{array}$ \\
\hline 1 & 35467 & 47975 & $57^{\prime} 33^{\prime \prime}$ & 1 h04'28' \\
\hline 2 & 16200 & 14189 & $25^{\prime} 25^{\prime \prime}$ & $19^{\prime} 22^{\prime \prime}$ \\
\hline 3 & 8950 & 8219 & $12^{\prime} 08^{\prime \prime}$ & $10^{\prime} 22^{\prime \prime}$ \\
\hline 4 & 15982 & 16312 & $23^{\prime} 12^{\prime \prime}$ & $21^{\prime} 57^{\prime \prime}$ \\
\hline 5 & 762 & 5834 & $2^{\prime} 36^{\prime \prime}$ & $8^{\prime} 00^{\prime \prime}$ \\
\hline 6 & 20050 & - & $27^{\prime} 31^{\prime \prime}$ & - \\
\hline 7 & 739 & - & $2^{\prime} 19^{\prime \prime}$ & - \\
\hline 8 & 1690 & - & $4^{\prime} 48^{\prime \prime}$ & - \\
\hline 9 & 559 & - & $1^{\prime} 45^{\prime \prime}$ & - \\
\hline 10 & 93 & - & $0^{\prime} 15^{\prime \prime}$ & -
\end{tabular}


The solutions themselves (without the header or footer information), including the five that were different from the solutions generated by Withall et al. (2008) are listed in Appendix A.

In relation to the results in Table 3 , it is important to note that comparing the results of different evolutionary systems is not straightforward and by no means definitive. Despite experiments being carried out on the same domain, a touch of luck is also involved in getting to a perfect solution quickly from evolutionary experiments. This is because, depending on the crossover of the genes and particularly on the (random) gene that gets chosen for mutation and the outcome of the mutation itself, a perfect solution may not be reached at the same time by different systems. Furthermore, even experiments carried out on the same system at different times, may not arrive at the same solution at the exact same generation. Keeping this in mind, Table 3 shows that Withall et al. (2008) need fewer generations than this research for two of the above solutions (2 and 3). This research needs fewer generations for solutions 1,4 and 5 .

One thing is evident however, that Withall's timings (time needed per generation) are lower than those in this research. As previously mentioned Withall's genetic evolutionary system was all written in Perl, thus there was no need for converting the evolved code to a Scripting language first to achieve dynamic execution, since Perl is already a scripting language. The system in this research however, was written in VB.NET and includes the additional step of forcing the execution of the solution as VBScript from within the VB.NET application. This affects the overall execution speed. Furthermore, it was observed during the experiment that VBScript displayed a message box to the user each time the script took longer than normal to execute. The user was then asked to choose whether to allow the script to continue running or end it and allow the system to move onto the next script.

We managed to change the VBScript control to make the decision by itself, without involving the user. Although this has sped up the execution, it is still an extra step that VBScript has to do behind the scenes, which increases the overall execution time for the evolutionary system.

A potential solution may be to change the system to execute the dynamic programs in Perl instead of VBScript and see if this makes a difference to the above timing issue. Another solution may be to allow .NET itself to execute the dynamic code through the inbuilt System.CodeDom.Compiler, however, this is a more complicated solution, which may still result in time wastage due to the additional manipulation that VB.NET will have to make to itself to compile the dynamic code at runtime.

\section{CONCLUSIONS}

This paper has discussed an innovative approach to mapping genotypes to phenotypes through XML rules in relation to software statements and structures as well as complete software programs. Utilising XML gives this technique many advantages including: improved readability, compatibility with many programming languages, portability and extendibility (XML is not restricted to a limited set of keywords defined by the proprietary vendors, which aids the process of creating rules of different levels of complexity).

Experiments were set up to test the complete evolutionary system on the evolution of 'Sorting' programs. The 'Sorting' program was chosen because it represents a well known, standard Computer Science problem, which is complex enough to really test the evolutionary system.

Our evolutionary system was originally created to deal with the evolution of Regular Expressions. However, it was discovered, that it was easily adaptable to other domains, including that of Complete Software Structures. In fact, the only two areas that needed to be changed to make the system work for the new domain, were the XML rules and the Fitness function.

The experimental results from the evolution of 'Sorting' programs highlight the efficacy and flexibility of our system, despite the complexity of the 'Sorting' problem for GP systems.

However, further testing needs to be done to ensure the reliability of this approach for other complex programs.

\section{ACKNOWLEDGEMENTS}

We would like to thank the team at ATM for their support. Also, thank you to ATM, CICE and Loughborough University for funding our work. We would also like to thank Daniel Sills for his help with some technical .NET concepts.

\section{REFERENCES}

Aho, A.V. \& Ullman, J.D. 1979. Principles of Compiler Design. Addison Wesley, ISBN 0-201-00022-9.

Atkinson-Abutridy, J., Mellish, C., Aitken, S. 2004. Combining information extraction with genetic algorithms for text mining. IEEE Intelligent Systems. 19(3), pp. 22-30.

Banzhaf, W. 1994. Genotype-Phenotype-Mapping and Neutral Variation - A case study in Genetic 
Programming. Proceedings of the International Conference on Evolutionary Computation. SpringerVerlag, pp.322-332.

Banzhaf, W. 2006. genotype-phenotype-mapping and neutral variation - A case study in Genetic Programming. Lecture Notes in Computer Science, Springer Berlin, 866, pp. 322-332.

Barrero, D., Camacho, D. \& R-Moreno, M. 2009. Automatic Web Data Extraction Based on Genetic Algorithms and Regular Expressions. Data Mining and Multi-agent Integration. Springer-Verlag, pp. 143.

Clarke, M., Hinde, C.J., Withall, M.S., Jackson, T.W., Philips, I.W., Brown, S. \& Watson, R. 2009. Allocating Railway Platforms using a Genetic Algorithm. Research and Development in Intelligent Systems XXVI. Springer London, pp. 421-434.

Conrad, E. 2007. Detecting Spam with Genetic Regular Expressions. SANS Institute Reading Room. Available: http://www.giac.org/certified_professionals/practicals/ GCIA/00793.php.

Dyer, J. \& Bentley, P. 2002. PLANTWORLD: Population Dynamics in Contrasting Environments. In Cantu-Paz E., GECCO, pp. 122-129.

Grosan, C. \& Abraham, A. 2008. Evolving Computer Programs for Knowledge Discovery. Social Science Research Network (SSRN).

Hsu, P-H. 2007. Feature extraction of hyperspectral images using wavelet and matching pursuit. ISPRS Journal of Photogrammetry and Remote Sensing. Elsevier Science, Amsterdam, 62 (2), pp. 78-92.

Jackson, D. 2005. Evolving Defence Strategies by Genetic Programming. In Lecture Notes in Computer Science. Springer Berlin, 3447, 281-290.

Keaveney, D. \& O'Riordan, C. 2009. Evolving Robust Strategies for an Abstract Real-time Strategy Game. Proceedings of the $5^{\text {th }}$ International Conference on Computational Intelligence and Games. pp. 371-378.

Keller, R.E. \& Banzhaf, W. 1996. Genetic Programming using Genotype-Phenotype Mapping from Linear Genomes into Linear Phenotypes. Proceedings of the First Annual Conference on Genetic Programming, California. pp. 116-122.

Klank, U., Padoy, N., Feussner, H. and Navab, N. 2008. Automatic feature generation in endoscopic images. International Journal of Computer Assisted Radiology and Surgery. Springer, 3, pp. 331-339.

Koza, J.R. 1992. Genetic Programming: On the Programming of Computers by Means of Natural Selection. MIT Press.

Langdon, W., Poli, R., McPhee, N. \& Koza, J.R. 2008. Genetic Programming: An Introduction and Tutorial with a Survey of Techniques and Applications. In Studies in Computational Intelligence. Springer, Berlin, 115, pp. 927-1028.

Moore, J.P. 2000. Exploring and Exploiting Models of the Fitness Landscape: A Case against Evolutionary Optimization. PhD Thesis, University of Plymouth.

O’Neill, M., Brabazon T., Ryan, C. \& Collins J.J. 2001. Developing a Market Timing System using Grammatical Evolution. Proceedings of GECCO.
Rothlauf, F. 2006. Representations for Genetic and Evolutionary Algorithms. Springer-Verlag New York.

Snajder, J., Basic, B.D., Petrovic, S. \& Sikiric, I. 2008. Evolving new lexical association measures using genetic programming. Proceedings of the Association for Computational Linguistics. Ohio, pp. 181-184.

Whigham, P.A. 1995. Grammatically-based Genetic Programming. Workshop on Genetic Programming.

Withall, M.S., Hinde, C.J. \& Stone, R.G. 2008. An improved representation for evolving programs. Journal of Genetic Programming and Evolvable Machines. Springer Netherlands, 10(1), pp. 37-70.

Xhemali, D., Hinde, C.J. \& Stone, R.G. 2009-a. DomainIndependent Genotype to Phenotype Mapping through XML Rules. International Journal of Computer Science Issues, 7(3).

Xhemali, D., Hinde, C.J. \& Stone, R.G. 2010-b. Genetic Evolution of Regular Expressions for the Automated Extraction of Course Names from the Web. Proceedings of the International Conference on Genetic and Evolutionary Methods. Las Vegas.

Yosif, N., Gramm, J., Wang, Q., Noble, W., Karp, R. \& Sharan, R. 2010. Prediction of Phenotype Information from Genotype Data. Communications in Information and systems. 10(2), pp. 99-114.

\section{APPENDIX A}

These are the 'Sorting' programs generated in this research. Solution (1) was shown in Figure 4. Solutions (1-5) match those obtained by Withall et al. (2008), whereas the remaining (6-10) are new.

Note that parts of the following programs may look different to 'hand written' code for 'Sorting' programs. Solutions (3) and (9) are the closest to the conventional 'hand coded' version.

\section{'Sorting' Solution: (2)}

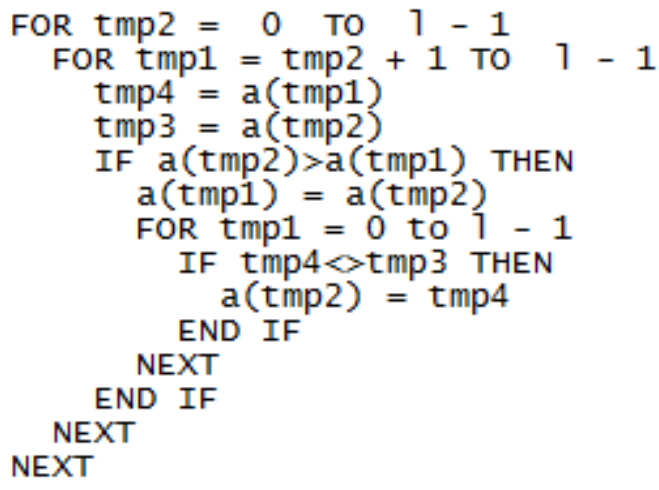


'Sorting' Solution: (3)

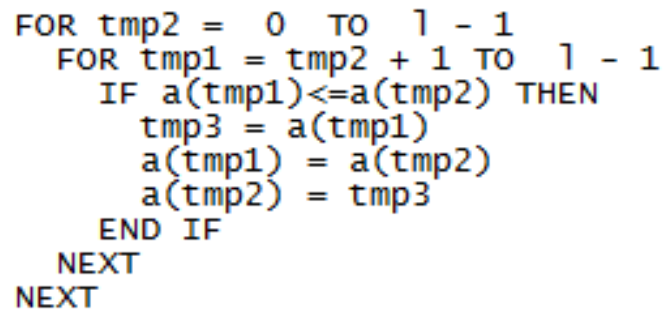

'Sorting' Solution: (4)

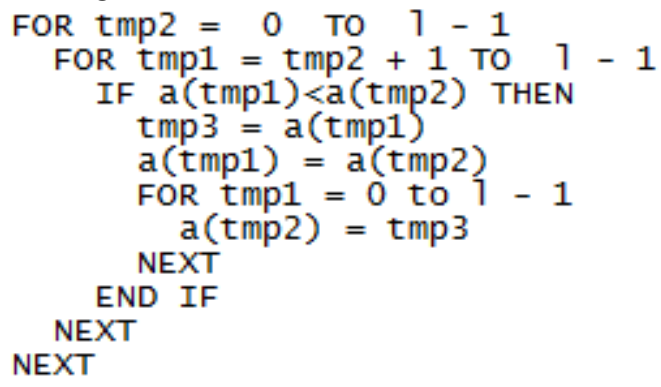

'Sorting' Solution: (5)

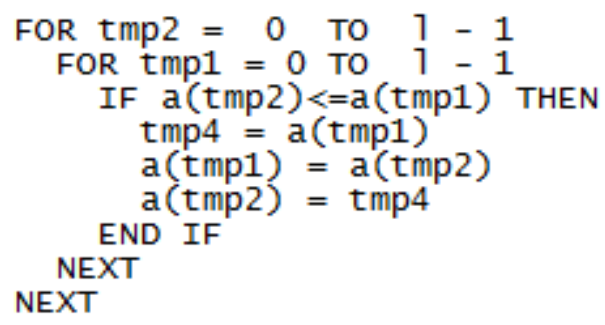

'Sorting' Solution: (6)

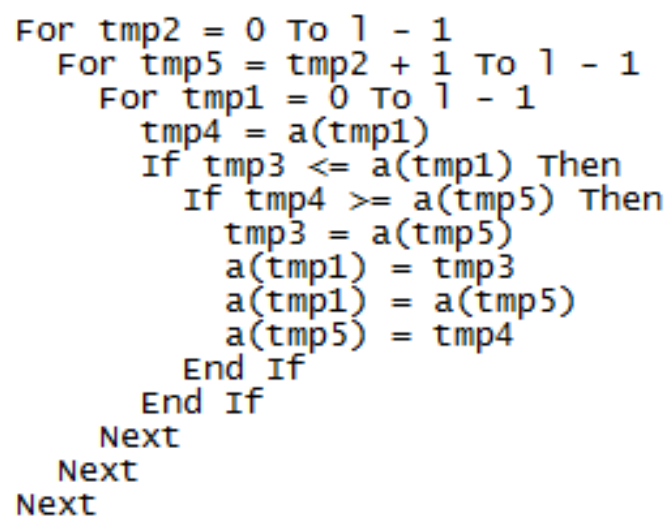

‘Sorting' Solution: (7)

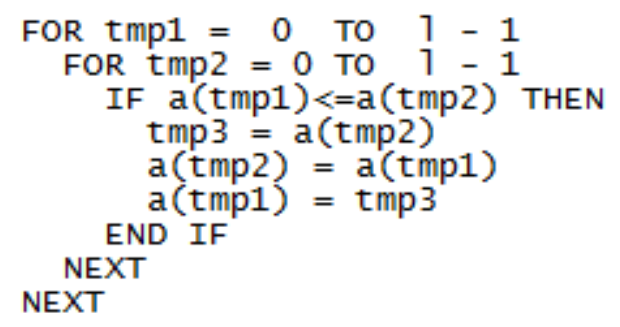

'Sorting' Solution: (8)

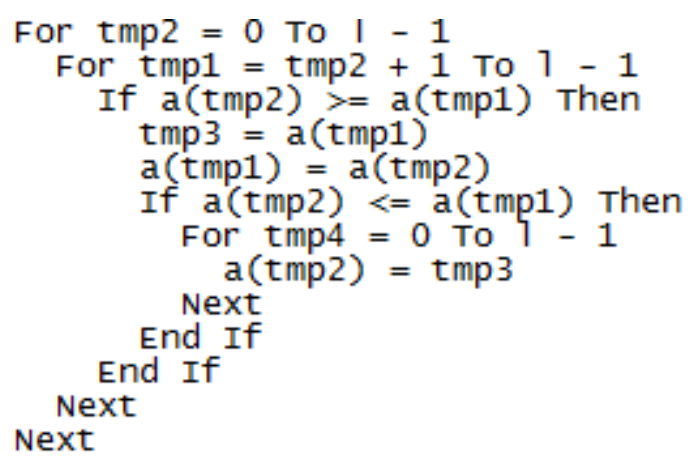

'Sorting' Solution: (9)

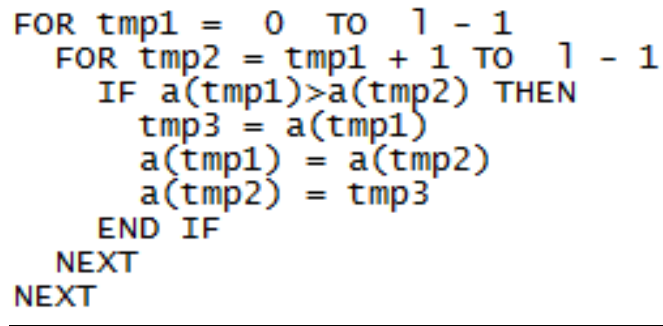

'Sorting' Solution: (10)

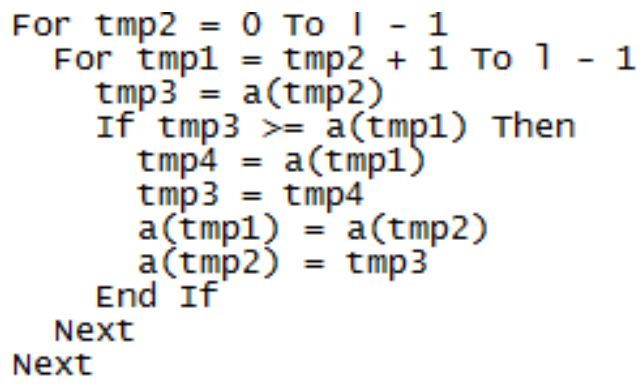

